\title{
Pathology-free third molars: assessing the perceived risk of future pathology
}

\author{
Perceived risk of future pathology associated with pathology-free third molars: a comparison of oral and maxillofacial \\ surgeons and family dentists O. Kostopoulou, M. R. Brickley, J. P. Shepherd, and R. G. Newcombe Br Dent J 2000; \\ 188: $28-31$
}

\section{Objective}

To examine and compare practitioners' judgements of risk of future pathology associated with pathology-free disease asymptomatic third molars.

\section{Subjects}

10 oral and maxillofacial surgeons and 18 family dentists $(90 \%$ male) with experience ranging from 5-28 years.

\section{Method}

Participants were presented with periapical radiographs of 36 asymptomatic, disease-free mandibular third molars and were informed of the age and sex of the patients and the degree of eruption of the third molars. Participants were asked to assess likelihood of future pathology in general, and more specifically, likelihood of root resorption, pericoronitis, periodontitis, cystic change and neoplasia if the third molar was left in situ.

\section{Results}

There was significant variation between the 28 raters but not between the two groups. Excepting assessment of future cystic change, there was no evidence that oral and maxillofacial surgeons and family dentists rated the 36 cases in consistently different ways.

\section{Conclusions}

Practitioners varied very considerably in their judgment of the risks of pathology associated with asymptomatic disease-free third molars. Specialisation did not account for this variation.

\section{Comment}

This paper, from a group experienced in third molar epidemiology and management, aimed to compare oral and maxillofacial surgeons (OMFS) and family dentists (FD) in their judgement of the risk of future disease associated with pathology-free and asymptomatic third molars (M3).

The results suggested little overall difference in assessment by the two groups, but it was interesting that FDs perceived a higher liklihood of cystic change than the OMFSs. It has been interesting to note in the literature that although earlier figures suggested an incidence of cystic change between $2-11 \%$, a recent study of apparently disease-free follicular tissue showed cyst formation in $37 \%$ of lower M3s. ${ }^{1}$

International professional debate has continued for many years about the risks of pathology developing in association with impacted but symptom-free M3. There were variations in rates of surgical removal and in earlier years a higher rate of what has been termed often inappropriately, 'prophylactic' removal. The picture came into focus with the publication in 1980 of the results of a consensus conference in the USA which included broad guidelines of indications for surgery. ${ }^{2}$ In the UK, both the Government and medical insurers began to show a greater interest in the subject. Indeed the Department of Health (DH) funded the largest audit project of its kind. ${ }^{3}$ This showed that most $(78 \%)$ of M3s removed were in fact associated with symptoms. The Faculty of Dental Surgery of the Royal College of Surgeons of England with whom the British Association of Oral \& Maxillofacial Surgeons had collaborated in the audit were then commissioned by the DH to develop detailed guidelines supported by the most robust evidence available. These were published in 1997 and highlighted the absence of any long-term randomised controlled trial results which compared the outcome of early surgery with a policy of review and intervention only in the event of development of pathology. ${ }^{4,5}$ A 4 -year study of this type is now running in the USA with the prospect of extension to UK patient cohorts in the next year or so.

The results of this multicentre trial are awaited with interest. It is hoped that they will help clinicians, commissioning authorities, NICE, most important, our patients to balance the potential benefits and complications of early surgery against the risks of pathology emerging in later years (which may necessitate intervention under less favourable conditions of acute infection and co-morbidity).

\section{J C Lowry}

Consultant Oral \& Maxillofacial Surgeon, Royal Bolton Hospital/Blackburn Royal Infirmary

1 Glosser J W, Campbell J H. Pathologic change in soft tissues associated with radiographically 'normal' third molar impactions. Br J Oral Maxillofac Surg 1999; 37: 259-260.

2 National Institute of Health. Consensus development conference for removal of third molars. J Oral Surg 1980; 38: 235-236.

3 Worrall S F, Riden K, Haskell R, Corrigan A M. UK National Third Molar Audit project; the initial report. Br J Oral Maxillofac Surg 1998; 36: 14-18.

4 Faculty of Dental Surgery. National Clinical Guidelines 1997. The Royal College of Surgeons of England. September 1997.

5 Current clinical practice and parameters of care. The management of patients with third molar teeth. The Faculty of Dental Surgery of the Royal College of Surgeons of England. September 1997. 